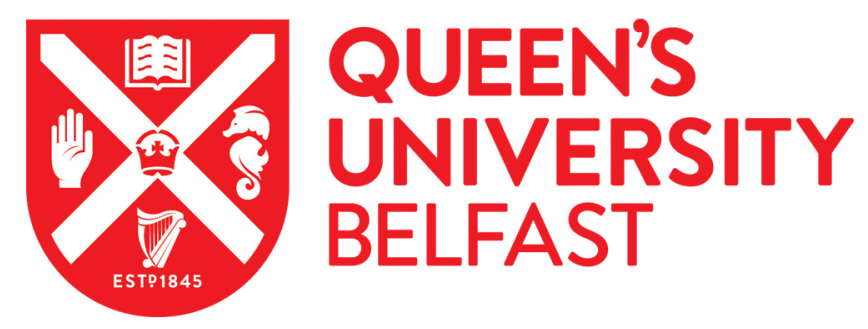

\title{
Identification and target-modifications of temporin-PE: A novel antimicrobial peptide in the defensive skin secretions of the edible frog, Pelophylax kl. esculentus
}

Sang, M., Wu, Q., Xi, X., Ma, C., Wang, L., Zhou, M., Burrows, J., \& Chen, T. (2018). Identification and targetmodifications of temporin-PE: A novel antimicrobial peptide in the defensive skin secretions of the edible frog, Pelophylax kl. esculentus. Biochemical and Biophysical Research Communications, 495(4), 2539-2546. https://doi.org/10.1016/j.bbrc.2017.11.173

\section{Published in:}

Biochemical and Biophysical Research Communications

\section{Document Version:}

Peer reviewed version

Queen's University Belfast - Research Portal:

Link to publication record in Queen's University Belfast Research Portal

\author{
Publisher rights \\ Copyright 2018 Elsevier. \\ This manuscript is distributed under a Creative Commons Attribution-NonCommercial-NoDerivs License \\ (https://creativecommons.org/licenses/by-nc-nd/4.0/), which permits distribution and reproduction for non-commercial purposes, provided the \\ author and source are cited.
}

\section{General rights}

Copyright for the publications made accessible via the Queen's University Belfast Research Portal is retained by the author(s) and / or other copyright owners and it is a condition of accessing these publications that users recognise and abide by the legal requirements associated with these rights.

Take down policy

The Research Portal is Queen's institutional repository that provides access to Queen's research output. Every effort has been made to ensure that content in the Research Portal does not infringe any person's rights, or applicable UK laws. If you discover content in the Research Portal that you believe breaches copyright or violates any law, please contact openaccess@qub.ac.uk. 
Identification and Target-modifications of Temporin-PE: A Novel Antimicrobial Peptide in the Defensive Skin Secretions of the Edible Frog, Pelophylax kl. esculentus

Mengru Sang ${ }^{1,2}$, Qinan $\mathrm{Wu}^{1, *}$, Xinping $\mathrm{Xi}^{2,}{ }^{*}$, Chengbang $\mathrm{Ma}^{2}$, Lei Wang ${ }^{2}$, Mei Zhou ${ }^{2}$, James F. Burrows $^{2}$, Tianbao Chen ${ }^{2}$

${ }^{1}$ School of Pharmacy, Nanjing University of Chinese Medicine; Collaborative Innovation Centre of Chinese Medicinal Resources Industrialization; National and Local Collaborative Engineering Centre of Chinese Medicinal Resources Industrialization and Formulae Innovative Medicine, Nanjing, Jiangsu, 210023, China

${ }^{2}$ School of Pharmacy, Queen’s University, Belfast BT9 7BL, Northern Ireland, UK

*Corresponding authors. Email: qnwyjs@163.com (Q. Wu); x.xi@qub.ac.uk (X. Xi); Tel: +86 (0)25 85811010 (Q. Wu); +44 (0)28 90971673 (X. Xi)

\begin{abstract}
A potent natural antimicrobial peptide named temporin-PE was identified and encoded from the skin secretions of Pelophylax kl. esculentus via "shotgun" cloning and LC-MS/MS fragmentation analysis. Target-modifications were carried out to further enhance the antimicrobial and anti-proliferative bioactivities, whilst decreasing the hemolytic effect. A range of bioassays demonstrated that replacing a proline with a tyrosine residue resulted in a loss of the bioactivity against Gram-negative bacteria, but dramatically improved the hemolytic and anti-proliferative activity, indicating the FLP- motif influences the hemolytic activity of temporins. Moreover, the coupling of TAT to the peptide dramatically improved its antimicrobial activity, indicating coupling TAT to these peptides could be considered as a potential tool to improve their antimicrobial activity. Overall, we have shown that targeted modifications of this natural antimicrobial peptide can adjust its bioactivities to help its development as an antibiotic or anti-proliferative agent.
\end{abstract}

Abbreviations: AMP, antimicrobial peptide; GM, the geometric mean; TI, the therapeutic index 
Keywords: temporin; peptide design; cytotoxicity; antimicrobial

\section{Introduction}

Anurans are a group of morphologically specialized amphibians widely distributed around the world. P. kl. esculentus is a common species of frog in Europe, where it is also known as the edible frog. Although P. kl. esculentus, previously known as "Rana esculenta", is a hybrid of the pool frog (Rana lessonae) and the marsh frog (Rana ridibunda), it possesses superior skin peptide immune defenses. Compared to the parental species, this hybrid species not only produces the most diverse peptides, but the antibiotic activity and stability of its antimicrobial peptides (AMPs) are also superior [1]. Therefore, the innate immune defense system of $P$. kl. esculentus is worthy of detailed research.

To date, more than thirty different AMPs have been discovered from the edible frog [2-9], most of which are brevinins $[4,6-8]$, esculentins $[4]$ and temporins $[5,6,9,10]$. Besides, there are also protease inhibitors [11] and bradykinins [12] in the skin secretions of $P$. kl. esculentus. According to the literature, temporins are a group of AMPs with potential to be developed as novel antibiotic agents due to their ability to kill microorganisms, including antibiotic-resistance bacteria. Temporins are short, C-terminally $\alpha$-amidated, highly hydrophobic and weakly charged or non-charged [13]. Their secondary structures are usually linear and $\alpha$-helical, which is one of the critical elements contributing to their cytotoxic effects upon microbial and cancer cells, due to their ability to destabilize membranes. Although, it is common for some AMPs to exhibit activity against cancer cells, and temporins have been shown to permeabilize several cell types, there are few reports about the impact of temporins on cancer cells, indeed, only the antimicrobial temporin-1CEa and temporin-La have been shown to exert significant activity against cancer cells [14-17]. The mechanism by which temporins impact upon cancer cells has been shown to relate to intracellular mechanisms involving mitochondria, including $\mathrm{Ca}^{2+}$ leakage, the collapse of mitochondrial membrane potential and over-generation of reactive oxygen species and membrane-denaturation due to the electrostatic interaction between the cationic peptides and anionic cell membrane $[14$, 17]. 


\section{Materials and Methods}

\section{Specimen Biodata and Harvesting of Skin Secretion}

The frogs were obtained, fed and skin secretions were harvested from the edible frog as described previously [9]. These procedures were subject to ethical approval and carried out under appropriate UK animal research personal and project licenses.

\section{Molecular Cloning of Temporin-PE Precursor-Encoding cDNA}

The "shotgun" cloning was carried out as previously described [9]. A degenerated primer (5'-GAWYYAYYHRAGCCYAAADATGTTCA-3') (W = A or T; Y = C or T; H = A or C or T; R $=\mathrm{A}$ or $\mathrm{G} ; \mathrm{D}=\mathrm{A}$ or $\mathrm{G}$ or $\mathrm{T}$ ) was subjected to 3 '-RACE reaction, from which the products were cloned and subsequently sequenced.

\section{Identification of Temporin-PE in the skin secretion}

The skin secretion was fractionated by RP-HPLC and all fractions were subjected to MALDI-TOF mass spectrometer. Then the fraction containing the same molecular weight of the putative mature peptide was analyzed by MS/MS fragmentation sequencing. The experimental procedures for RP-HPLC, MALDI-TOF and LC-MS/MS have been described previously [18].

\section{Design and Chemical Synthesis of Peptides}

Here, the natural-occurring temporin-PE was employed as a template for the targeted design. The temporins present in P. kl. esculentus are structurally similar to peptides discovered in wasp venom, which display a conserved N-terminal FLP motif which caused hemolysis [19]. Therefore, to determine if this motif was causing a similar impact in temporin-PE, we modified the FLP motif to produce temporin-PEa and examined its hemolytic activity. Particularly, substituting Pro $^{3}$ by $\mathrm{Tyr}^{3}$, designed by the genetic algorithm (GA) mutation in HeliQuest tool, was used to change the backbone of temporin-PE but remained the similar physicochemical parameters, namely temporin-PEa. Meanwhile, another analog was designed by coupling a TAT (-GRKKRRQRRR-) at the C-terminus of temporin-PE, namely temporin- $\mathrm{PEb}$, in order to enhance the cell penetrating 
activity as well as investigate the potential intracellular targets of temporin-PE [20]. All peptides were chemically-synthesized by solid phase Fmoc chemistry [18].

\section{Predicted Physicochemical Parameters, and Secondary Structures of Peptides}

The secondary structure predictions and models were processed using the I-TASSER server [21-24]. The helical wheels and the physiochemical parameters of peptides were predicted using the HeliQuest tool $[25,26]$. The secondary structures of all peptides were examined by circular dichroism (CD) and analyzed by the K2D3 web server as described before [18].

\section{Antimicrobial Assay}

The minimal inhibitory concentrations (MICs) of peptides were evaluated by the standard microdilution assay described previously [18]. Six different microorganism strains, Candida albicans (NCPF 1467), Staphylococcus aureus (NCTC 10788), Escherichia coli (NCTC 10418), Methicillin-resistant S. aureus (MRSA) (NCTC 12493), Enterococcus faecalis (NCTC12697) and Pseudomonas aeruginosa (ATCC 27853), were utilized in this assay. The geometric mean (GM) of MICs for each peptide to inhibit the growth of C. albicans, S. aureus, E. coli, MRSA, E. faecalis and $P$. aeruginosa, was calculated to estimate the overall antimicrobial activity of peptides. The therapeutic index (TI) of the peptide was shown as the ratio of $\mathrm{HC}_{50}$ for erythrocytes to the $\mathrm{GM}$. The $\mathrm{HC}_{50}$ here is the concentration of a peptide inducing $50 \%$ erythrocyte hemolysis.

\section{Time-killing assay}

The time killing assay was performed following the Corinne's protocol with modifications [27]. Briefly, S. aureus (NCTC 10788) and MRSA (NCTC 12493) were employed in this assay. $4 \mu \mathrm{M}, 2 \mu \mathrm{M}$ and $1 \mu \mathrm{M}$ of each peptide were applied for S. aureus and $8 \mu \mathrm{M}, 4 \mu \mathrm{M}$ and $2 \mu \mathrm{M}$ were applied for MRSA. Appropriate time points were selected, as indicated, to observe the viable counts.

\section{Anti-proliferation assay}


The anti-proliferation assay was performed using MTT as in our previous studies [28, 29]. The non-small cell lung cancer cell line NCI-H157, human neuronal glioblastoma cell line U251MG, human prostate carcinoma cell line PC-3, melanoma cell line MDA-MB-435s and human microvessel endothelial cells HMEC-1 were utilized in this assay. The GMs for anti-proliferative activity were calculated. The TI value of each peptide was shown as the ratio of $\mathrm{IC}_{50}$ for HMEC-1 cells to the GM for four types of cancer cells.

\section{Hemolysis Assay}

The hemolysis bioactivity of the three peptides against defibrinated horse erythrocytes was determined following the same protocol as used previously [11, 28]. 1\% TritonX-100 was employed as the positive control.

\section{Statistical Analysis}

All the bioactivity assays had five independent replicates. Data were subjected to statistical analysis using Prism (Version 5.0, U.S.A). The one-way ANOVA was used to calculate the significance of the sample set. Error bars in the charts indicate standard error of the mean.

\section{Results}

\section{Molecular cloning of A Novel Precursor-encoding cDNA}

A nucleotide and translated amino acid sequence of an open-reading frame (ORF) encoding a potential antimicrobial peptide precursor were obtained via shotgun cloning (Figure 1). The petide consists of 62 amino acid residues, divided into five definite domains including a highly conserved putative signal peptide region containing 23 amino acid residues, an acidic amino acid residue-rich "spacer" region, a typical -Lys-Arg- propeptide convertase processing site, a putative mature peptide region containing 13 amino acid residues, namely temporin-PE, and a C-terminal -Gly-Lyspropeptide which acts as an amide donor to C-terminal amindation of the mature peptide. The nucleotide sequence of temporin-PE has been deposited to GenBank under the accession code MF957260. 


\section{Confirmation of the Primary Structure of Temporin-PE}

Abundant peaks were fractionated from the skin secretions of $P$. kl. esculentus by RP-HPLC (Figure 2A). MALDI-TOF confirmed the peak at 152 min was the fraction containing the predicted mature peptide (Figure 2C) and this this fraction was further analyzed via MS/MS fragmentation analysis (Figure 2B and Table 1).

\section{Secondary Structures and Physicochemical Properties of Temporin-PE, Temporin-PEa and Temporin-PEb}

Temporin-PE, Temporin-PEa and Temporin-PEb were all predicted to be typical $\alpha$-helical peptides (Figure 3A-C). The predicted helical wheel structures of these peptides demonstrated that all three contained a hydrophobic face, exhibiting that higher amphipathy of temporin-PEb than which of the other two (Figure 3D-F). Temporin-PE and temporin-PEa displayed similar physicochemical properties, while temporin-PEb exhibited a larger $\alpha$-helical domain, higher net positive charges and hydrophilicity (Table 2). CD spectra of the peptides confirmed that the peptides were random coil but formed $\alpha$-helical structures in the membrane-mimic environment (Figure 3G\&H). Although the actual contents of $\alpha$-helical domains of each peptide are lower than the predicted ones, it reveals that the temporin-PEb possesses the largest $\alpha$-helical domain, whereas temporin-PEa contained the smallest.

\section{Antimicrobial assay}

Temporin-PE potently inhibited the growth of the pathogenic fungi (C. albicans), the Gram-positive bacteria S. aureus and MRSA, but showed lower efficacy against the Gram-negative bacteria such as E. faecalis and P. aeruginosa. Temporin-PEa exerted similar bioactivity except against the Gram-negative bacteria, E. coli and P. aeruginosa (Table 3). However, temporin-PEb showed the most potent antimicrobial bioactivity against all six microorganisms.

\section{Time-killing kinetics assay}


Temporin-PE and temporin-PEb showed concentration-dependent and time-dependent bactericidal activity against $S$. aureus and MRSA, however, temporin-PEa did not impact upon these two strains at all tested concentrations (Figure 4A-F). Temporin-PEb was the most potent AMP starting to kill S. aureus at concentrations from $1 \mu \mathrm{M}$, while taking 30 minutes to kill MRSA at concentrations from $4 \mu \mathrm{M}$. Temporin-PE displayed potent activity against S. aureus at $8 \mu \mathrm{M}$, but was much less potent against MRSA.

\section{Cancer cell viability assay}

Since temporin-PE and the two designed analogs exerted cytotoxicity to pathogenic microorganisms and erythrocytes, the ability of them functioning to cancer cells were also studied. The result indicated that two designed peptides possessed more efficient anti-proliferative activity than temporin-PE (Figure 4G-K). However, unlike temporin-PEa without seriously cytotoxicity on normal human microvessel endothelial cells HMEC-1 at the $10 \mu \mathrm{M}$, temporin-PEb showed nonspecific cytotoxicity towards all tested cell lines. Temporin-PEa displayed the relatively highest TI values than the other peptides (Table 4).

\section{Hemolysis assay}

Temporin-PE, temporin-PEa and temporin-PEb exhibited different degrees of hemolysis on horse erythrocytes with $\mathrm{HC}_{50}$ of $39.64 \mu \mathrm{M}, 531.7 \mu \mathrm{M}$ and $44.64 \mu \mathrm{M}$, respectively. Temporin-PEa and temporin-PEb possessed lower hemolytic activity than temporin-PE, especially temporin-PEa, with 13-fold less hemolytic activity.

\section{Discussion}

To date, except temporin-PE, only five temporins have been isolated from P. kl. esculentus [5, $6,9,10]$. According to the literature, temporin-PE possesses potent antimicrobial activity which is 25-fold, 4-fold and 2.5-fold stronger than the best inhibitory effects of other reported temporins in P. kl. esculentus against C. albicans, S. aureus and E. coli respectively (Table 5). It is noteworthy that most temporin members have the common motif FLP- at their N-termini and a leucine at the 
second position near their C-termini, as well approximately 70\% hydrophobic residues, which make them highly hydrophobic [30]. Compared to temporin-1Ec and temporin-1Ee, temporin-PE exhibited a more potent antimicrobial activity, something which could be explained by the position of the positive charge in its sequence. Temporin-PE, containing a lysine residue at position $7\left(\right.$ Lys $\left.^{7}\right)$, possesses a more potent antimicrobial effect, especially against the growth of fungi, as $\mathrm{Lys}^{7}$ is located on the hydrophilic face of the helix domain, while a lysine residue at position 11 might not be on the hydrophilic face unless the helical domain extended to the C-terminus [31]. We therefore presumed that the lysine located on the hydrophobic region or random domain could not play roles as well as that on the hydrophilic region of temporins especially in the field of electrostatic interactions and bindings with negatively charged residues in the cell membranes.

Temporin-PEa retained its antimicrobial activity except which was against Gram-negative bacterium. Both peptides share the similar secondary structure and physicochemical properties relating to antimicrobial activity, which suggested the significance of Pro on membrane interaction between peptides and Gram-negative bacterium membrane. Usually, AMPs firstly bind lipopolysaccharide (LPS) micelles when they interact with Gram-negative bacterium. Such binding might lead some AMPs to translocate through the outer membrane of Gram-negative bacterium, as the magnesium and calcium ions that hold together LPS would replace metal ions, breaking the outer membrane, and resulting in facilitating entry of additional AMPs from the exterior [32]. Previous studies have shown that proline plays an essential role in outer membrane disruption and bacterial cell lysis activity, as the more proline residues present, the stronger the binding between AMPs and LPS [33]. Therefore, removing the proline might decrease the LPS binding affinity, meaning temporin-PEa could not overcome the LPS hurdle and the integrity of these bacterial cells would not be disrupted, resulting in a loss of activity against Gram-negative bacterium. Moreover, compared to tyrosine, proline, which usually forms a slight hinge structure, is more possible to make peptides more easily to fasten on the zwitterionic membrane. That is confirmed what we had anticipated, the temporin-PEa showed a vastly reduced hemolytic activity indicating this FLP motif is again important in these peptides. However, it increased the anti-proliferative activity against cancer cells. As it is reported, the plasma membrane surface of 
cancer cells contains abundant phosphatidylserines, glycoproteins, gangliosides and some other anionic molecules, some of which are often over-expressed in cancer cell lines [34]. That is presumably the reason why the substitution of proline with tyrosine leads to a decreased toxicity towards normal cells, but shows increased anti-proliferative activity toward cancer cells. We would therefore hypothesize this anti-proliferative activity results from the phenolic hydroxyl moieties binding to target molecules, such as sialic acids of the O-glycoproteins, N-glycoproteins and gangliosides, which are overexpressed on cancer cells.

TAT is an arginine-rich decapeptide derived from HIV-1, an ideal cell-penetration segment to transit drugs through cell membranes via membrane translocation and endocytosis, with no hemolytic effects, mild antibiotic activity and inhibits the growth of fungi with MICs ranging from $3 \mu \mathrm{M}$ to $24 \mu \mathrm{M}$ [35]. We hypothesized that the electrostatic forces between temporin-PEb and anionic cell membranes would become much stronger when eight positive charges were introduced as part of TAT. Compared to temporin-PE, temporin-PEb demonstrated an 8-fold and 64-fold stronger antimicrobial effect against E. coli and P. aeruginosa respectively, 4-fold stronger against C. albicans and E. faecalis, and two-fold against S. aureus and MRSA. In addition, temporin- $\mathrm{PEb}$ also acted more rapidly against these microporganisms. Although the antimicrobial mechanism of temporin-PEb was not verified experimentally, it is presumed that the addition of TAT improved the electrostatic interaction, as well as helping the peptide to penetrate into the cytoplasm to interfere the biological processes such as DNA replication and protein synthesis, leading to its markedly improved antimicrobial activity [36]. Meanwhile, the anti-proliferative activity of temporin-PEb was also increased against all the cell lines examined. So we hypothesize that temporin-PEb might not only disrupt the plasma membrane through the permeabilization, but also involve the intracellular targets, such as mitochondrial membrane, after the penetrating the cell membrane by TAT.

In summary, this study has identified a novel temporin which shows potent antimicrobial activity. We then designed target-modifications to enhance the antimicrobial and anti-proliferative bioactivities of this peptide, whilst decreasing its hemolytic effect. The substitutions made demonstrated that the FLP motif, as previously shown for other similar peptides, is associated with the hemolytic activity of temporin-PE. In addition, modifying the FLP motif also decreased the 
potency of this peptide against Gram-negative bacteria, while enhancing its anti-proliferative activity. Furthermore, this work shows that introducing a TAT peptide at the C-terminus of temporin dramatically increased the antimicrobial activity of temporin-PE. Therefore, this study has opened up new perspectives on how modifying natural AMPs can alter their bioactivities.

\section{Acknowledgments}

This work was supported by Ministry of Science and Technology [grant number 2015FY111500]; National Natural Science Foundation China [grant number 81773854]; and Technology Research Funds of State Administration of Traditional Chinese Medicine [grant number 201407002]. Besides, Mengru Sang was in receipt of a one-year scholarship from the China Scholarship Council.

\section{References}

[1] J.M. Daum, L.R. Davis, L. Bigler, D.C. Woodhams, Hybrid advantage in skin peptide immune defenses of water frogs (Pelophylax esculentus) at risk from emerging pathogens, Infect Genet Evol, 12 (2012) 1854-1864.

[2] M.L. Mangoni, N. Papo, G. Mignogna, D. Andreu, Y. Shai, D. Barra, M. Simmaco, Ranacyclins, a New Family of Short Cyclic Antimicrobial Peptides: Biological Function, Mode of Action, and Parameters Involved in Target Specificity, Biochemistry, 42 (2003) 14023-14035.

[3] A.T. Lebedev, E. Damoc, A.A. Makarov, T.Y. Samgina, Discrimination of leucine and isoleucine in peptides sequencing with Orbitrap Fusion mass spectrometer, Analytical chemistry, 86 (2014) 7017-7022.

[4] M. Simmaco, G. Mignogna, D. Barra, F. Bossa, Antimicrobial peptides from skin secretions of Rana esculenta. Molecular cloning of cDNAs encoding esculentin and brevinins and isolation of new active peptides, Journal of Biological Chemistry, 269 (1994) 11956-11961.

[5] M. Simmaco, G. Mignogna, D. Barra, F. Bossa, Novel antimicrobial peptides from skin secretion of the European frog Rana esculenta, FEBS letters, 324 (1993) 159-161.

[6] M.F. Ali, F.C. Knoop, H. Vaudry, J.M. Conlon, Characterization of novel antimicrobial peptides from the skins of frogs of the Rana esculenta complex, Peptides, 24 (2003) 955-961.

[7] Y. Wang, F.C. Knoop, I. Remy-Jouet, C. Delarue, H. Vaudry, J.M. Conlon, Antimicrobial peptides of the brevinin-2 family isolated from gastric tissue of the frog, Rana esculenta, Biochemical and biophysical research communications, 253 (1998) 600-603.

[8] X. Chen, H. Wang, L. Wang, M. Zhou, T. Chen, C. Shaw, Identification of Miscellaneous Peptides from the Skin Secretion of the European Edible Frog, Pelophylax kl. Esculentus, The protein journal, 35 (2016) 291-299.

[9] X. Chen, H. Wang, M. Yang, L. Wang, M. Zhou, T. Chen, C. Shaw, Identification and bioactivity 
evaluation of two novel temporins from the skin secretion of the European edible frog, Pelophylax kl. esculentus, Biochem Biophys Res Commun, 476 (2016) 566-573.

[10] M. Simmaco, D. De Biase, C. Severini, M. Aita, G. Falconieri Erspamer, D. Barra, F. Bossa, Purification and characterization of bioactive peptides from skin extracts of Rana esculenta, Biochimica et Biophysica Acta-General Subjects, 1033 (1990) 318-323.

[11] X. Chen, H. Wang, Y. Shen, L. Wang, M. Zhou, T. Chen, C. Shaw, Kunitzins: Prototypes of a new class of protease inhibitor from the skin secretions of European and Asian frogs, Biochem Biophys Res Commun, 477 (2016) 302-309.

[12] X. Chen, L. Wang, H. Wang, H. Chen, M. Zhou, T. Chen, C. Shaw, A fish bradykinin ( $\operatorname{Arg}^{0}, \operatorname{Trp}^{5}$, Leu $^{8}$-bradykinin) from the defensive skin secretion of the European edible frog, Pelophylax kl. esculentus: structural characterization; molecular cloning of skin kininogen cDNA and pharmacological effects on mammalian smooth muscle, Peptides, 32 (2011) 26-30.

[13] A.K. Mahalka, P.K. Kinnunen, Binding of amphipathic alpha-helical antimicrobial peptides to lipid membranes: lessons from temporins B and L, Biochim Biophys Acta, 1788 (2009) 1600-1609.

[14] C. Wang, Y. Zhou, S. Li, H. Li, L. Tian, H. Wang, D. Shang, Anticancer mechanisms of temporin-1CEa, an amphipathic $\alpha$-helical antimicrobial peptide, in Bcap-37 human breast cancer cells, Life Sci, 92 (2013) 1004-1014.

[15] Q.Z. Yang, C. Wang, L. Lang, Y. Zhou, H. Wang, D.J. Shang, Design of potent, non-toxic anticancer peptides based on the structure of the antimicrobial peptide, temporin-1CEa, Arch Pharm Res, 36 (2013) 1302-1310.

[16] C. Wang, H.B. Li, S. Li, L.L. Tian, D.J. Shang, Antitumor effects and cell selectivity of temporin-1CEa, an antimicrobial peptide from the skin secretions of the Chinese brown frog (Rana chensinensis), Biochimie, 94 (2012) 434-441.

[17] Y. Diao, W. Han, H. Zhao, S. Zhu, X. Liu, X. Feng, J. Gu, C. Yao, S. Liu, C. Sun, F. Pan, Designed synthetic analogs of the $\alpha$-helical peptide temporin-La with improved antitumor efficacies via charge modification and incorporation of the integrin $\alpha v \beta 3$ homing domain, J Pept Sci, 18 (2012) 476-486.

[18] Y. Gao, D. Wu, L. Wang, C. Lin, C. Ma, X. Xi, M. Zhou, J. Duan, O.R.P. Bininda-Emonds, T. Chen, C. Shaw, Targeted Modification of a Novel Amphibian Antimicrobial Peptide from Phyllomedusa tarsius to Enhance Its Activity against MRSA and Microbial Biofilm, Front Microbiol, 8 (2017) 628.

[19] M. Simmaco, G. Mignogna, S. Canofeni, R. Miele, M.L. Mangoni, D. Barra, Temporins, antimicrobial peptides from the European red frog Rana temporaria, The FEBS Journal, 242 (1996) 788-792.

[20] H. Brooks, B. Lebleu, E. Vivès. Tat peptide-mediated cellular delivery: back to basics, Adv. Drug Delivery Rev. 57 (2005) 559-77.

[21] A. Roy, A. Kucukural, Y. Zhang, I-TASSER: a unified platform for automated protein structure and function prediction, Nature protocols, 5 (2010) 725.

[22] J. Yang, R. Yan, A. Roy, D. Xu, J. Poisson, Y. Zhang, The I-TASSER Suite: protein structure and function prediction, Nature methods, 12 (2015) 7-8.

[23] Y. Zhang, I-TASSER server for protein 3D structure prediction, BMC bioinformatics, 9 (2008) 40.

[24] I-TASSER server. Available online: https://zhanglab.ccmb.med.umich.edu/I-TASSER/ (accessed on 12 October 2017)

[25] R. Gautier, D. Douguet, B. Antonny, G. Drin, HELIQUEST: a web server to screen sequences with specific $\alpha$-helical properties, Bioinformatics, 24 (2008) 2101-2102.

[26] HeliQuest Version 2. Available online: http://heliquest.ipmc.cnrs.fr/ (accessed on 11 
September 2017).

[27] C. Ruppen, P. Sendi, Time kill assays for Streptococcus agalactiae and synergy testing, (2015).

[28] X. Guo, C. Ma, Q. Du, R. Wei, L. Wang, M. Zhou, T. Chen, C. Shaw, Two peptides, TsAP-1 and TsAP-2, from the venom of the Brazilian yellow scorpion, Tityus serrulatus: evaluation of their antimicrobial and anticancer activities, Biochimie, 95 (2013) 1784-1794.

[29] R. Wang, Y. Zhou, T. Chen, M. Zhou, L. Wang, C. Shaw, Identification and functional analysis of a novel tryptophyllin peptide from the skin of the red-eye leaf frog, Agalychnis callidryas, Int J Biol Sci, 11 (2015) 209-219.

[30] D. Wade, A. Silveira, J. Silberring, P. Kuusela, H. Lankinen, Temporin antibiotic peptides: a review and derivation of a consensus sequence, Protein and Peptide Letters, 7 (2000) 349-358.

[31] F. Abbassi, C. Galanth, M. Amiche, K. Saito, C. Piesse, L. Zargarian, K. Hani, P. Nicolas, O. Lequin, A. Ladram, Solution structure and model membrane interactions of temporins-SH, antimicrobial peptides from amphibian skin. A NMR spectroscopy and differential scanning calorimetry study, Biochemistry, 47 (2008) 10513-10525.

[32] M. Zasloff, Antimicrobial peptides of multicellular organisms, nature, 415 (2002) 389-395.

[33] S. Bera, A. Ghosh, S. Sharma, T. Debnath, B. Giri, A. Bhunia, Probing the role of Proline in the antimicrobial activity and lipopolysaccharide binding of indolicidin, J Colloid Interface Sci, 452 (2015) 148-159.

[34] Y.Y. Han, H.Y. Liu, D.J. Han, X.C. Zong, S.Q. Zhang, Y.Q. Chen, Role of glycosylation in the anticancer activity of antibacterial peptides against breast cancer cells, Biochem Pharmacol, 86 (2013) 1254-1262.

[35] H.J. Jung, Y. Park, K.S. Hahm, D.G. Lee, Biological activity of Tat (47-58) peptide on human pathogenic fungi, Biochem Biophys Res Commun, 345 (2006) 222-228.

[36] K.A. Brogden, Antimicrobial peptides: pore formers or metabolic inhibitors in bacteria?, Nat Rev Microbiol, 3 (2005) 238-250.

\section{Legends to Figures and Tables}

Figure 1. Nucleotide and translated ORF amino acid sequences of the precursor cDNA encoding the peptide temporin-PE, from the skin secretions of $P$. kl. esculentus. The putative signal peptide sequence is single-underlined, the mature peptide sequence is double-underlined, and an asterisk indicates the stop codon.

Figure 2. (A) RP-HPLC chromatogram of skin secretion of RP-HPLC P. kl. esculentus at a wavelength of $214 \mathrm{~nm}$. The peak with an arrow is the peak of temporin-PE. (B) The annotated MS/MS spectrum of the RP-HPLC fraction of temporin-PE. (C) MALDI-TOF mass spectrum of the RP-HPLC fraction containing temporin-PE. 
Figure 3. Predicted secondary structure models of (A) temporin-PE (B) temporin-PEa, (C) temporin-PEb and helical wheel projections of (D) temporin-PE, (E) temporin-PEa, (F) temporin-PEb; CD spectra of temporin-PE and its analogs $(50 \mu \mathrm{M})(\mathrm{G})$ in $10 \mathrm{mM}$ ammonium acetate water solution and $(\mathrm{H})$ in $50 \% \mathrm{TFE} / 10 \mathrm{mM}$ ammonium acetate water solution. The arrows indicate the hydrophobic regions.

Figure 4. The time-killing kinetics of three peptides at different concentrations of (A) $4 \mu \mathrm{M}$, (B) $2 \mu \mathrm{M}$, (C) $1 \mu \mathrm{M}$ against S. aureus and (D) $8 \mu \mathrm{M}$, (E) $4 \mu \mathrm{M}$, (F) $2 \mu \mathrm{M}$ against MRSA; The cell viabilities of (G) NCI-H157 (H) U251MG (I) PC-3 (J) MDA-MB-435s (K) HMEC-1 and the hemolytic effects (L) after treatment of temporin-PE and its analogs

Table 1. Expected b- and y-ions arising from collision induced of the doubly-charged precursor ion. Actual fragment ions observed in MS/MS fragmentation are marked with the single-underlined bold typeface.

Table 2. Primary structures, predicted secondary structures and physicochemical properties of temporin-PE, temporin-PEa and temporin-PEb

Table 3. MICs, GMs and TI values of Temporin-PE, Temporin-PEa and Temporin-PEb against six microorganisms

Table 4. Impact of temporin-PE, temporin-PEa and temporin-PEb on cancer cell viability

Table 5. A comparison of the primary structures and MICs of temporins discovered in P. kl. esculentus 


\section{Figure 1.}

\begin{tabular}{lllllllllllllllll}
$M$ & $F$ & $T$ & $M$ & $K$ & $K$ & $S$ & L & L & L & L & $F$ & $F$ & L & $G$ & T & I \\
\hline
\end{tabular}

1 ATGTTCACCA TGAAGAAATC CCTGTTACTC CTTTTCTTCC TTGGAACCAT TACAAGTGGT ACTTCTTTAG GGACAATGAG GAAAAGAAG AACCTTGGTA $\begin{array}{lllllllllllllllll}N & L & S & L & C & E & E & E & R & D & T & D & E & E & E & R & R\end{array}$

51 CAACTTATCT CTCTGTGAGG AAGAGAGAGA TACTGATGAG GAAGAAAGAA GTTGAATAGA GAGACACTCC TTCTCTCTCT ATGACTACTC CTTCTTTCTT

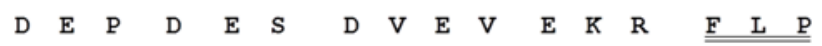

101 GAGATGAGCC GGATGAAAGT GATGTTGAAG TGGAAAAACG ATTTCTGCCG CTCTACTCG CCTACTTTCA CTACAACTTC ACCTTTTTGC TAAAGACGGC $\begin{array}{llllllllllllllllllll}I & V & A & K & \text { L } & \text { L } & \text { S } & G & \text { L } & \text { L } & G & K & \text { * }\end{array}$

151 ATTGTGGCAA AGCTGCTCTC AGGTTTGTTG GGAAAGTAAC CAAAAATGTT TAACACCGTT TCGACGAGAG TCCAAACAAC CCTTTCATTG GTTTTTACAA 201 GAAACTTGGC AACTGAATCT GTGGAAACAT CTGCATTGAA ATATAATTTA CTTTGAACCG TTGACTTAGA CACCTTTGTA GACGTAACTT TATATTAAAT

251 GCTAAATATC TAACAGATGT CTTATAAAAA AAATAAATAA ATATGTTACA CGATTTATAG ATTGTCTACA GAATATTTTT TTTATTTATT TATACAATGT

301 AAAAAAAAAA AAAAAAAAAA

TTTTTTTTTT TTTTTTTTTT 
Figure 2.

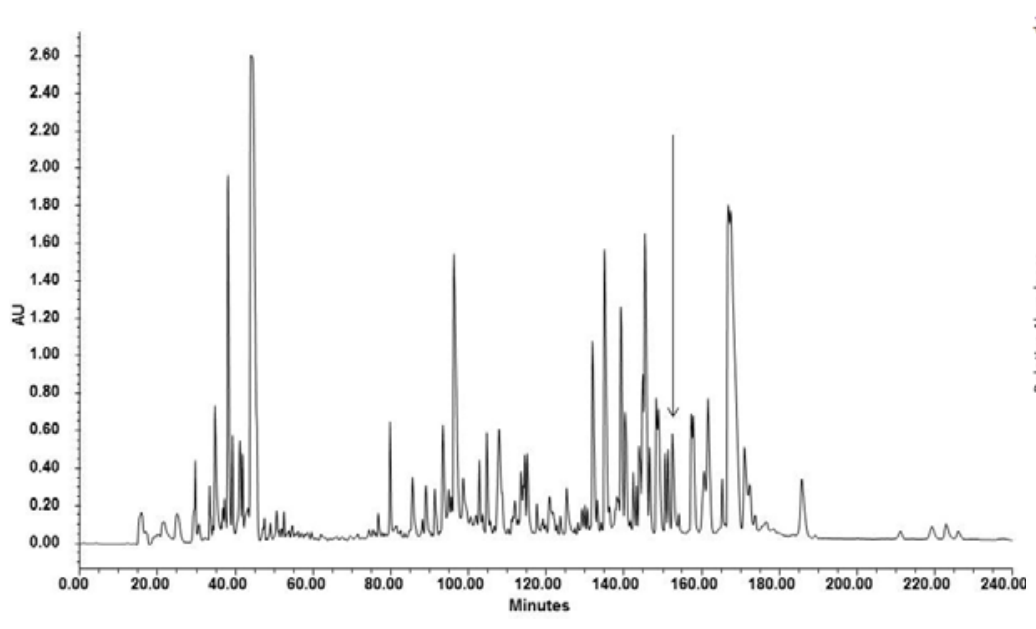

A

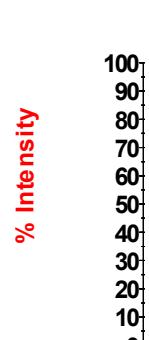

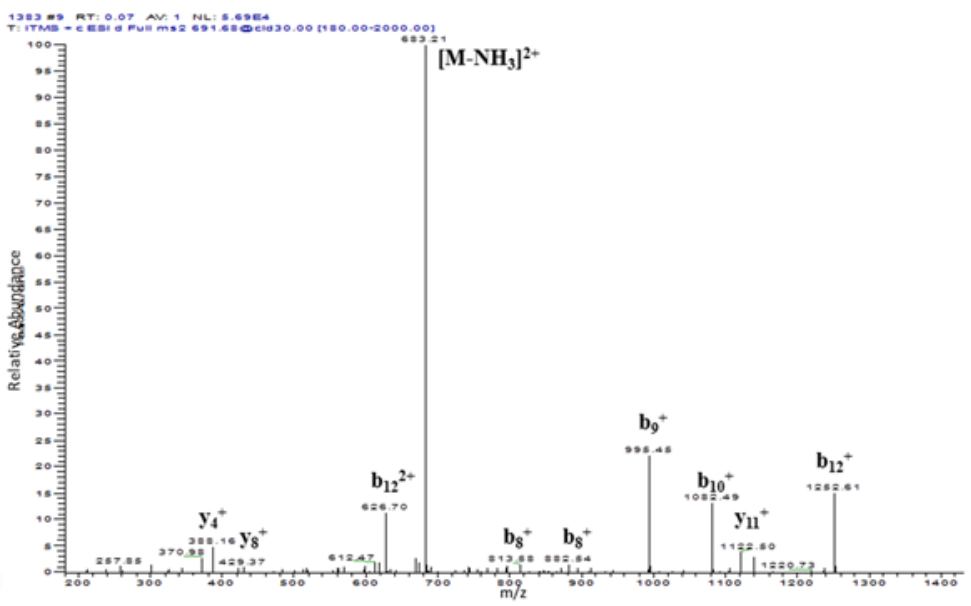

B

Voyager Spec \#1[BP = 1383.3, 57856]

1383.76

$5.6 E+4$

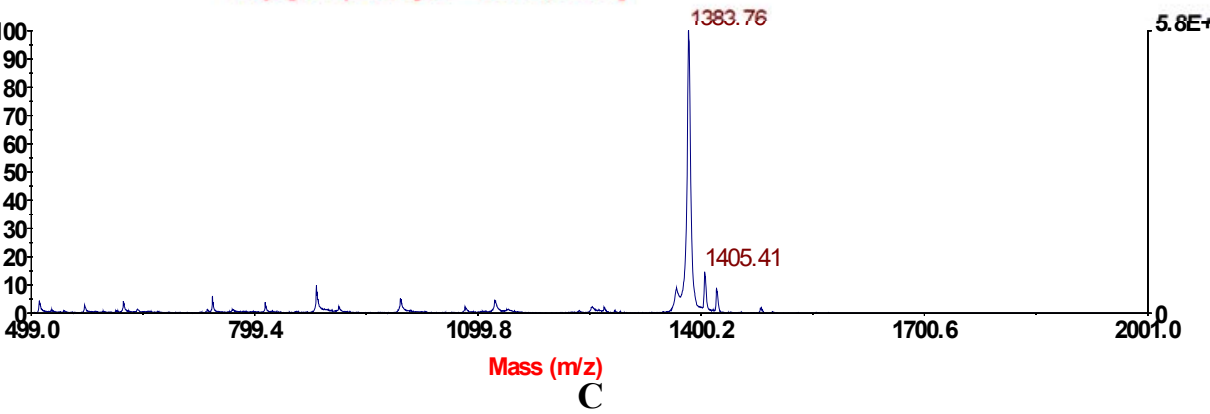


Figure 3.

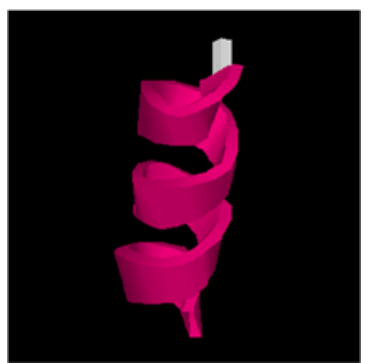

A

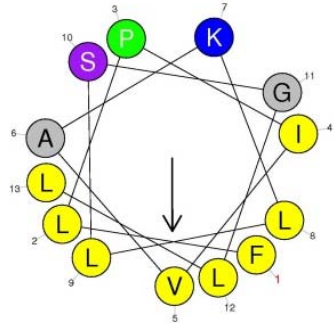

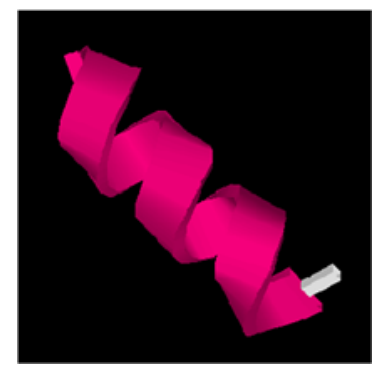

B

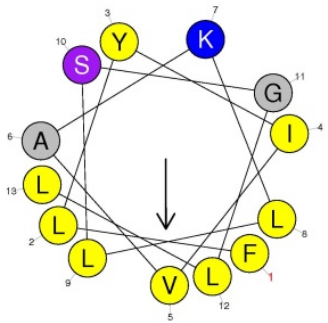

E

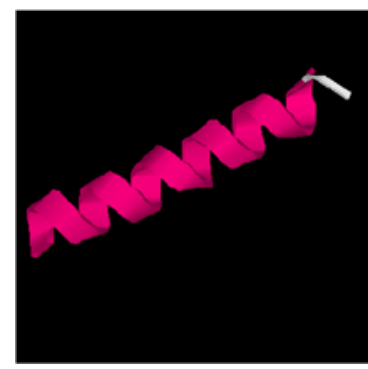

C
D

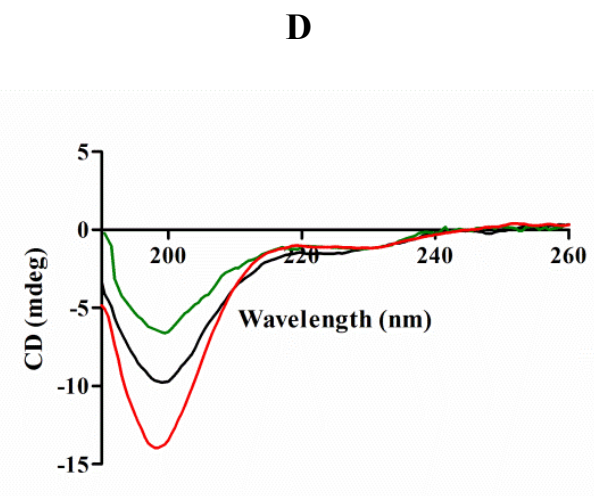

G

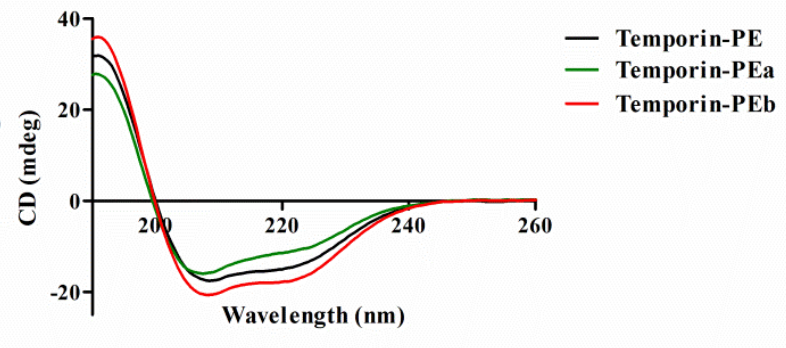

H 
Figure 4.
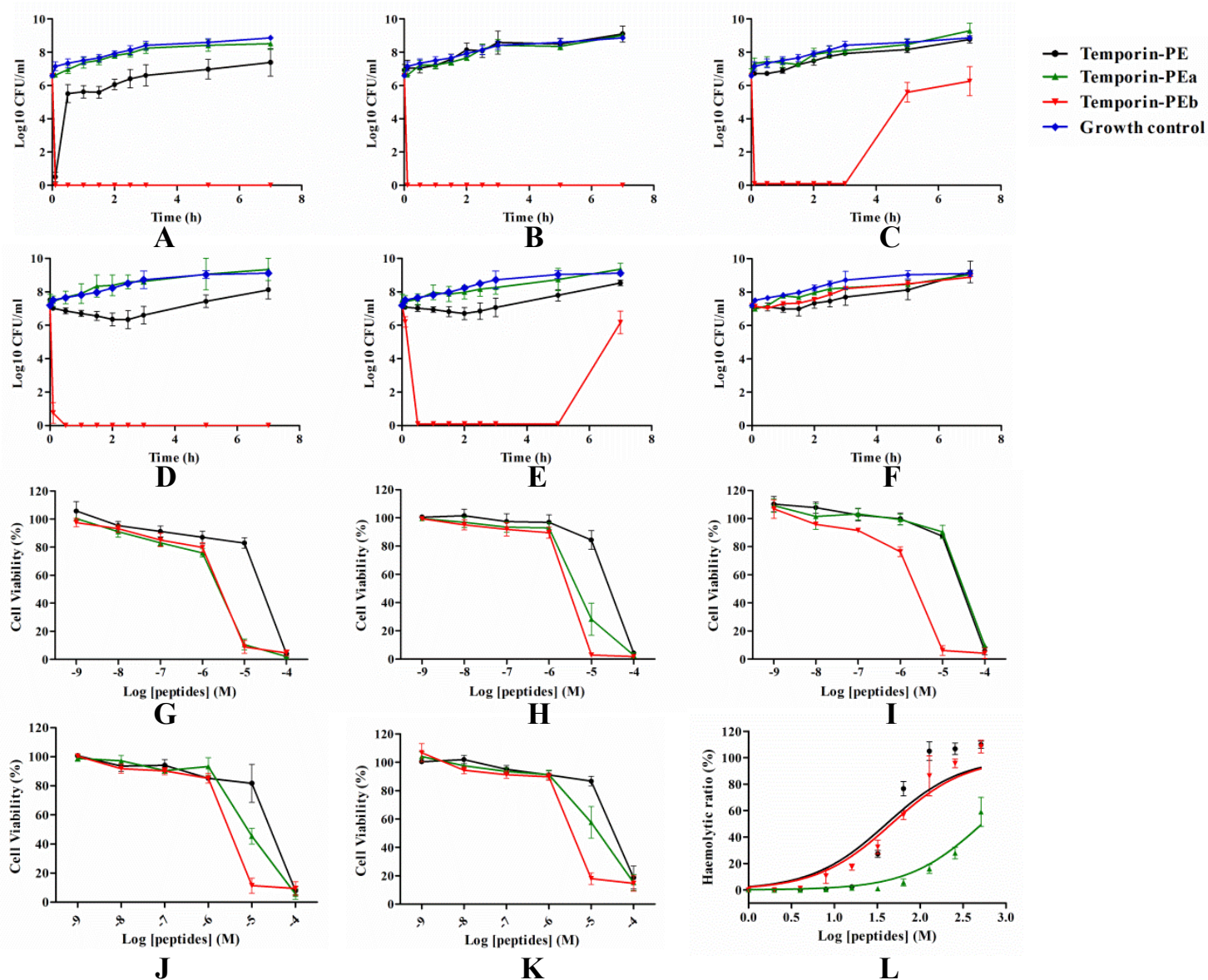

L 
Table 1.

\begin{tabular}{ccccccc}
\hline$\# \mathbf{1}$ & $\mathbf{b}(\mathbf{1 +}$ & $\mathbf{b}(\mathbf{2}+)$ & Seq. & $\mathbf{y}(\mathbf{1 +})$ & $\mathbf{y}(\mathbf{2 +})$ & $\# \mathbf{2}$ \\
\hline 1 & 148.0757 & 74.54149 & $\mathrm{~F}$ & & & 13 \\
2 & $\underline{\mathbf{2 6 1 . 1 5 9 7 7}}$ & 131.08352 & L & $\underline{\mathbf{1 2 3 5 . 8 4 5 0 3}}$ & $\underline{\mathbf{6 1 8 . 4 2 6 1 5}}$ & 12 \\
3 & 358.21254 & 179.60991 & P & $\underline{\mathbf{1 1 2 2 . 7 6 0 9 6}}$ & $\underline{\mathbf{5 6 1 . 8 8 4 1 2}}$ & 11 \\
4 & 471.29661 & 236.15194 & I & $\underline{\mathbf{1 0 2 5 . 7 0 8 1 9}}$ & $\underline{\mathbf{5 1 3 . 3 5 7 7 3}}$ & 10 \\
5 & $\underline{\mathbf{5 7 0 . 3 6 5 0 3}}$ & 285.68615 & V & $\underline{\mathbf{9 1 2 . 6 2 4 1 2}}$ & 456.8157 & 9 \\
6 & $\underline{\mathbf{6 4 1 . 4 0 2 1 5}}$ & 321.20471 & A & $\underline{\mathbf{8 1 3 . 5 5 5 7}}$ & 407.28149 & 8 \\
7 & $\underline{\mathbf{7 6 9 . 4 9 7 1 2}}$ & 385.2522 & K & $\underline{\mathbf{7 4 2 . 5 1 8 5 8}}$ & $\underline{\mathbf{3 7 1 . 7 6 2 9 3}}$ & 7 \\
8 & $\underline{\mathbf{8 8 2 . 5 8 1 1 9}}$ & 441.79423 & L & 614.42361 & 307.71544 & 6 \\
9 & $\underline{\mathbf{9 9 5 . 6 6 5 2 6}}$ & 498.33627 & L & $\underline{\mathbf{5 0 1 . 3 3 9 5 4}}$ & 251.17341 & 5 \\
10 & $\underline{\mathbf{1 0 8 2 . 6 9 7 2 9}}$ & 541.85228 & S & $\underline{\mathbf{3 8 8 . 2 5 5 4 7}}$ & 194.63137 & 4 \\
11 & $\underline{\mathbf{1 1 3 9 . 7 1 8 7 6}}$ & $\underline{\mathbf{5 7 0 . 3 6 3 0 2}}$ & G & $\underline{\mathbf{3 0 1 . 2 2 3 4 4}}$ & 151.11536 & 3 \\
12 & $\underline{\mathbf{1 2 5 2 . 8 0 2 8 3}}$ & $\underline{\mathbf{6 2 6 . 9 0 5 0 5}}$ & L & 244.20197 & 122.60462 & 2 \\
13 & & & L-Amidated & 131.1179 & 66.06259 & 1 \\
\hline
\end{tabular}

Table 2.

\begin{tabular}{cccc}
\hline & \multicolumn{2}{c}{ Peptides } \\
\cline { 2 - 4 } & Temporin-PE & Temporin-PEa & Temporin-PEb \\
\hline Sequence & FLPIVAKLLSGLLa & FLYIVAKLLSGLLa & FLPIVAKLLSGLLGRKKRRQRRR \\
Structure Prediction ${ }^{1}$ & CCHHHHHHHHCCC & CHHHHHHHHHCCC & CCHHHHHHHHHHHHHHHHHCC \\
Confident Score & 9127999975329 & 9613998872439 & 91389999998861068877329 \\
Actual $\alpha$-helix $(\%)$ & 47.65 & 40.37 & 55.43 \\
Nonpolar residues $(\%)$ & 76.92 & 76.92 & 43.48 \\
Hydrophobicity $(\mathrm{H})$ & 1.024 & 1.042 & 0.220 \\
Hydrophobic moment $(\mu \mathrm{H})$ & 0.570 & 0.552 & 0.304 \\
Net charge & +2 & +2 & +10
\end{tabular}

${ }^{1} \mathrm{C}$, Coil; H, Helix

Table 3.

\begin{tabular}{ccccccccc}
\hline \multirow{2}{*}{ Meptide } & $\begin{array}{c}\boldsymbol{C} \text {. } \\
\text { albicans }\end{array}$ & $\begin{array}{c}\boldsymbol{S} \text { M. } \\
\text { aureus }\end{array}$ & E. coli & MRSA & $\begin{array}{c}\boldsymbol{E} \text {. } \\
\text { faecalis }\end{array}$ & $\begin{array}{c}\boldsymbol{P} \text {. } \\
\text { aeruginosa }\end{array}$ & GM $(\boldsymbol{\mu M})$ & TI \\
\hline temporin-PE & 4 & 2 & 16 & 4 & 8 & 128 & 8.98 & 4.41 \\
temporin-PEa & 2 & 1 & $>512$ & 2 & 2 & $>512$ & 10.08 & 52.2 \\
temporin-PEb & 1 & 1 & 2 & 2 & 2 & 2 & 1.59 & 28.0 \\
\hline
\end{tabular}

Table 4.

\begin{tabular}{ccccc}
\hline & \multirow{2}{*}{ Cell line } & \multicolumn{3}{c}{ Peptide } \\
\cline { 3 - 5 } & NCI-H157 & temporin-PE & temporin-PEa & temporin-PEb \\
\hline \multirow{3}{*}{$\mathrm{IC}_{50}(\mu \mathrm{M})$} & $\mathrm{U} 251 \mathrm{MG}$ & 34.56 & 3.398 & 3.464 \\
& $\mathrm{PC}-3$ & 25.13 & 3.520 & 3.087 \\
& $\mathrm{MDA}-\mathrm{MB}-435 \mathrm{~s}$ & 38.56 & 27.62 & 3.051 \\
& $\mathrm{HMEC}-1$ & 33.23 & 9.864 & 3.483 \\
& & 58.62 & 40.30 & 9.976 \\
\hline $\mathrm{GM}(\mu \mathrm{M})$ & 32.48 & 7.555 & 3.265 \\
\hline $\mathrm{TI}$ & 1.805 & 5.334 & 3.055 \\
\hline
\end{tabular}

NC, not calculated

Table 5.

$\begin{array}{llllll}\text { Peptide } & \text { sequence } & \text { Hydrophobicity } & \text { Hydrophobic } & \text { Net } & \text { MIC }(\mu \mathrm{M})\end{array}$




\begin{tabular}{clcccccc} 
& & $(\mathbf{H})$ & moment $(\boldsymbol{\mu H})$ & charge & $\begin{array}{c}\boldsymbol{C} \text {. } \\
\text { albicans }\end{array}$ & $\begin{array}{c}\boldsymbol{S} \text {. } \\
\text { aureus }\end{array}$ & $\begin{array}{c}\boldsymbol{E} \text { c. } \\
\text { coli }\end{array}$ \\
\hline temporin-PE & FLPIVAKLLSGLLa & 1.024 & 0.570 & +2 & 4 & 2 & 16 \\
temporin-1Ec & FLPVIAGLLSKLFa & 1.031 & 0.598 & +2 & $>160$ & 10 & 40 \\
temporin-1Ee & FLPVIAGVLSKLFa & 0.994 & 0.586 & +2 & $>100$ & 8 & $>100$ \\
temporin-1Re & FLPGLLA-- -GLLa & 1.132 & 0.507 & +1 & $>160$ & 60 & $>160$ \\
A1 & FLPAIAGILSQLFa & 1.028 & 0.587 & +1 & $\mathrm{ND}^{1}$ & $>200$ & $>50$ \\
B9 & FLPLIAGLLGKLFa & 1.071 & 0.585 & +2 & $\mathrm{ND}$ & $\mathrm{ND}$ & $\mathrm{ND}$ \\
\hline
\end{tabular}

${ }^{1} \mathrm{ND}$, not determined 